\title{
HOX genes in ovarian cancer
}

\author{
Zoë L Kelly', Agnieszka Michael ${ }^{1}$, Simon Butler-Manuel², Hardev S Pandha ${ }^{1}$ and Richard GL Morgan ${ }^{1 *}$
}

\begin{abstract}
The HOX genes are a family of homeodomain-containing transcription factors that determine cellular identity during development. Here we review a number of recent studies showing that HOX genes are strongly expressed in ovarian cancer, and that in some cases the expression of specific HOX genes is sufficient to confer a particular identity and phenotype upon cancer cells. We also review the recent advances in elucidating the different functions of HOX genes in ovarian cancer. A literature search was performed using the search terms HOX genes (including specific HOX genes), ovarian cancer and oncogenesis. Articles were accessed through searches performed in ISI Web of Knowledge, PubMed and ScienceDirect. Taken together, these studies have shown that HOX genes play a role in the oncogenesis of ovarian cancer and function in the inhibition of apoptosis, DNA repair and enhanced cell motility. The function of HOX genes in ovarian cancer oncogenesis supports their potential role as prognostic and diagnostic markers, and as therapeutic targets in this disease.
\end{abstract}

Keywords: Ovarian, Cancer, HOX, Therapy, HXR9

\section{Introduction}

The $H O X$ genes constitute a family of transcription factors that play key roles in embryonic development, especially in the patterning of the anterior to posterior axis, from the level of the hindbrain to the end of the spine. They are characterized by a highly conserved homeodomain region consisting of a 61-amino acid motif which enables HOX proteins to bind to specific regions of DNA in order to transcriptionally activate or repress target genes. HOX proteins can function as monomers or homodimers, although their target DNA binding affinities and specificities are enhanced by functioning as heterodimers or heterotrimers with members of the three-amino acid loop extension (TALE) family of cofactors, PBX and MEIS. HOX genes play fundamental roles during embryonic development, controlling anterior-posterior pattern formation, proliferation and differentiation [1]. In adult tissue, $H O X$ genes have been implicated in a variety of biological pathways including homeostasis, cell differentiation and the maintenance of organ functioning [2].

In mammals, 39 HOX genes have been identified and organised into 4 paralogous clusters (A, B, C and D) located on 4 different chromosomes [3]. Each cluster

\footnotetext{
* Correspondence: r.morgan@surrey.ac.uk

${ }^{1}$ Postgraduate Medical School, Faculty of Health and Medical Sciences, University of Surrey, GU2 7WG, UK

Full list of author information is available at the end of the article
}

contains 9-11 genes aligning in 13 paralogue groups based on homeobox sequence similarity and the position within a cluster. In embryonic development each $H O X$ gene is expressed in a spatiotemporal pattern whereby the position of the $H O X$ gene within a $H O X$ cluster corresponds to its order of expression along the anterior to posterior axis. Thus for example, the 5' genes (paralogues 9-13) are involved in the differentiation of genitourinary structures in the lumbosacral region. $H O X$ expression in adult tissues often reflects embryonic expression, and different cancers show distinct changes from normal adult expression [4]. These include temporospatial deregulation where $H O X$ gene expression in a tumour of a specific tissue is temporospatially different from the expression seen in the normal tissue, and/or the increased expression of $H O X$ genes that are usually expressed at lower levels in the normal tissue. In addition, epigenetic changes can result in the down-regulation or silencing of certain $H O X$ genes which normally function as tumour suppressors. Numerous studies have demonstrated deregulated $H O X$ gene expression in cancer including lung, prostate, breast, colon, bladder and thyroid cancer [5-9] and also in ovarian cancer.

\section{HOX genes and Ovarian Cancer}

During development of the female reproductive system four HOX genes, HOXA9, HOXA10, HOXA11, and HOXA13 are expressed uniformly along the Müllerian 
duct axis, although in adults their expression becomes spatially restricted to particular organs. HOXA9 becomes expressed in the fallopian tubes, HOXA10 is expressed in the developing uterus, HOXA11 in the lower uterine segment and cervix and HOXA13 in the upper vagina [10]. Their expression is thought to be important in preserving a high level of developmental plasticity of the female reproductive system due to the dramatic structural and functional changes which occur during the oestrous cycle and pregnancy [10]. It is thought that inappropriate expression of these genes is an early step in epithelial ovarian neoplasia as they induce aberrant epithelial differentiation. A study by Cheng et al. (2005) [11] used immunohistochemical analysis of biopsied tissue to show that the HOX genes which normally regulate Müllerian duct differentiation were not expressed in normal ovarian surface epithelia (OSE) but were expressed in epithelial ovarian cancers (EOCs). The $H O X$ expression pattern appears to determine the histological identity of EOCs with serous papillary, endometriod and mucinous tumours showing overexpression of HOXA9, HOXA10 and HOXA11, respectively [11]. Another $H O X$ gene, $H O X A 7$, was reported to be aberrantly expressed both at the RNA level (by quantitative PCR) and at the protein level (by immunohistochemistry using an anti-HOXA7 antibody) in ovarian cancer tissues which display Müllerian-like characteristics, however little or no expression was found in undifferentiated ovarian carcinomas and normal OSE [12]. When expressed ectopically in an undifferentiated ovarian mouse tumour, HOXA7 was shown to promote the ability of HOXA9, HOXA10 and HOXA11 to induce Müllerian differentiation rather than induce lineage specificity itself $[11,12]$. These results suggest $H O X A 7$ plays a role in epithelial differentiation of OSE in ovarian epithelial cancers, although Ota et al. (2007) found HOXA7 overexpression in all ovarian carcinomas tested, including undifferentiated subtypes. This could suggest that HOXA7 is associated with Müllerian differentiation but is not sufficient to maintain it (Figure 1).

Several other studies have reported altered $H O X$ gene expression in ovarian carcinomas when comparing to normal OSE [12-14] however, there are some discrepancies. Naora et al. (2001) [15] found HOXB7 was expressed at higher levels in ovarian carcinomas compared to normal OSE. This was supported by a later study by Yamashita et al. (2006) [13] who created an expression profile of HOX genes in ovarian-derived samples. In this study overexpression of $16 \mathrm{HOX}$ genes in ovarian cancer cell lines were found, the most common being HOXB7, HOXA13 and HOXB13. Overexpression varied between cell lines but of these 16 genes,

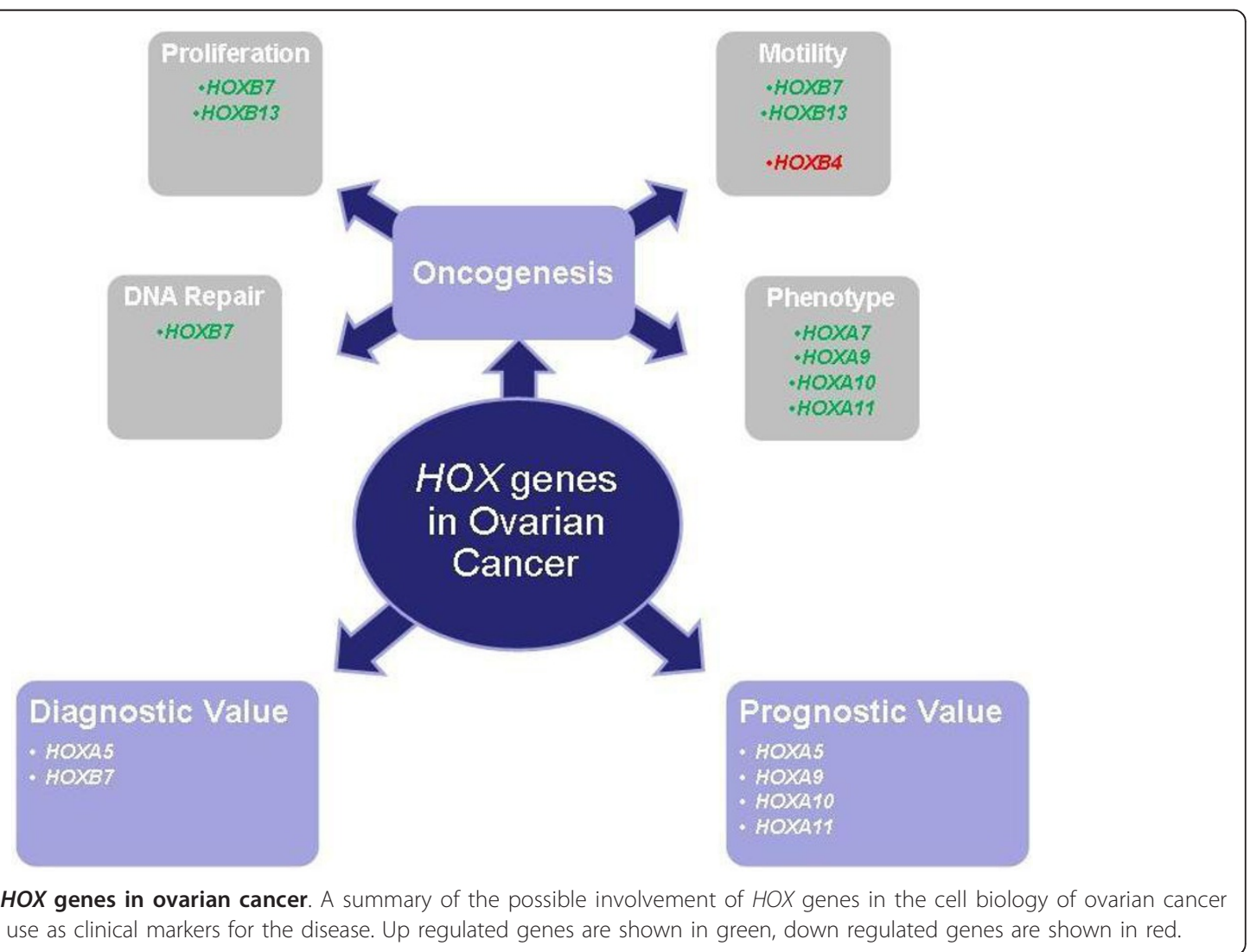

Figure 1 Role of HOX genes in ovarian cancer. A summary of the possible involvement of HOX genes in the cell biology of ovarian cancer 
HOXA10, A13, B4, B7, B13 and C13 showed little or no expression in normal samples (Table 1). It should be noted though that both of these studies examined the expression of HOX genes at the RNA level only by quantitative PCR.

Slightly different results were found in a more recent study by Hong et al. (2010) [16]. In this study the expression of $36 \mathrm{HOX}$ genes in ovarian cancer cell lines and tissues were compared to normal ovarian tissue, revealing a difference in expression of 11 HOX genes (HOXA7, B3, B4, B6, C10, C11, D1, D3, D10, D11 and $D 13)$. Of these 11 genes, $H O X B 4$ was the only $H O X$ gene showing significantly higher levels of expression in ovarian cancer cell lines than in normal ovarian tissue $(\mathrm{p}=0.029)$. Its expression was confirmed at protein level by Western blot analysis, with exclusive expression in all 4 ovarian cancer cell lines and all 7 ovarian cancer tissue samples and not in the normal ovarian tissues. HOXB4 has also been implicated as a cancer-related gene in other malignancies including breast cancer, leukaemia, osteosarcoma and lung cancer [17-20].

\section{Function of HOX genes in ovarian cancer Tumour Growth}

In addition to phenotypic determination, $H O X$ genes are thought to play a role in the oncogenesis of ovarian cancer. HOXB7 overexpression in immortalised OSE cells was shown to upregulate basic fibroblast growth factor (bFGF). bFGF is a potent mitogenic and angiogenic

Table 1 Over expression of HOX genes in ovarian cancer cell lines using RT-PCR

\begin{tabular}{llllll}
\hline \multicolumn{5}{c}{ Cell Line } \\
\hline HOX gene & SKOV-3 & CaOV3 & JHOC-6 & SMOV2 & ES-2 \\
\hline A3 & - & - & - & 2.0 & - \\
A4 & - & - & - & - & 2.0 \\
A7 & - & 2.1 & - & - & - \\
A10 & 12.1 & 13.3 & 3.5 & - & 12.6 \\
A13 & $>300$ & $>300$ & $>300$ & $>300$ & $>300$ \\
\hline B2 & 9.2 & - & - & - & 4.1 \\
B3 & 6.6 & - & - & - & - \\
B4 & 267 & 44.9 & - & 141.8 & 91.8 \\
B5 & 14.9 & - & - & - & - \\
B6 & 9.4 & - & - & - & - \\
B7 & $>300$ & $>300$ & $>300$ & $>300$ & $>300$ \\
B8 & 47.6 & - & 3.2 & - & - \\
B9 & 6.1 & 3.1 & 3.0 & & 5.6 \\
B13 & $>300$ & $>300$ & $>300$ & $>300$ & $>300$ \\
\hline C13 & - & 3.3 & - & - & - \\
\hline D13 & 3.6 & - & - & - & - \\
\hline Sh0 & & & & & - \\
\hline
\end{tabular}

Shows over expression of HOX genes in ovarian cancer cell lines by RT-PCR. Numbers indicate folds of expression level of each HOX gene in cancer cells compared with that in normal samples. After ref [13]. factor, although in this study it should be noted that the majority (95\%) of bFGF protein was intracellular and a relatively limited amount may therefore be available for cell to cell signalling [15]. HOXB13 has also been shown to enhance the proliferation of ovarian cancer cell lines SKOV-3 and OVCAR5 in vivo, and to promote the growth of a mouse ovarian cancer cell line in vivo and in vitro [21]. This oncogenic function of $H O X B 13$ is thought to require activated ras, as $H O X B 13$ promoted tumourgenesis in ovarian cancer cell lines containing genetic alterations in $p 53, m y c$ and $K$-ras but not in cell lines containing genetic alterations in $p 53, m y c$ and $A k t$. In this ras activated cell line, HOXB13 also conferred resistance to tamoxifen-mediated apoptosis suggesting a pro-survival role in ovarian cancer.

HOXA10 is also strongly expressed at the protein level in ovarian clear cell adenoarcinomas (OCCA, 20/29 cases) $[11,22]$ but not expressed in normal ovarian epithelia, ovarian serous adenocarcinomas, or endometrial cysts [22]. When over expressed in the human OCCA derived cell line ES-2, HOXA10 resulted in increased proliferation, and a two-fold increase in cell invasion as measured by a Matrigel invasion assay. Correspondingly, its expression in tumour samples correlates with poor survival. The 5-year survival rate in patients with $H O X A 10$ positive tumours was only $30 \%$, but it was $55.6 \%$ in the HOXA10 negative group [22].

\section{Cell motility and spreading}

In addition to promoting proliferation, $H O X B 7$ and $H O X B 13$ are also thought to be associated with the invasive characteristics of ovarian cancer cells. This invasive ability was investigated in a study by Yamashita et al. (2006) [13] using the invasive cancer cell line SKOV-3, which overexpresses HOXB7 and HOXB13. Antisense $H O X B 7$ and $H O X B 13$ fragments were introduced into SKOV-3 cells which results in an $85 \%$ and $50 \%$ reduction of motility, respectively, suggesting a role in cancer cell invasion. However, as invasion was not completely suppressed the function of these $H O X$ genes may be redundant and complemented by closely related genes such as HOXA13, which was also overexpressed in this cell line.

Invasive EOC cell lines also show overexpression of HOXA4 compared to non-invasive cell lines, suggesting a possible role for HOXA4 in promoting migration and invasion. However a number of studies have indicated that the role of HOXA4 could be invasion-suppressive as siRNA-mediated knockdown of HOXA4 enhanced cellular motility in normal OSE cells treated with EGF [23], although it had no affect on the basal levels of migration in the absence of EGF. This finding was supported in part by Klausen et al. (2009) [24] where knockdown of HOXA4 in OVCAR-3 and OVCAR-8 cells increased migration (although not Matrigel 
invasion). HOXA4 knockdown also reduced cell-cell adhesion and $\beta 1$ integrin protein level within cell colonies, suggesting $\beta 1$ integrin has a role in mediating these changes. Intriguingly, these changes in protein level are not reflected at the RNA level, indicating that the effect of HOXA4 on $\beta 1$ integrin and hence cell motility may be through an indirect mechanism. Taken together these findings suggest that HOXA4 is indeed primarily a suppressor of invasion, and it is possible then that the increased HOXA4 expression observed in invasive cell lines may be linked to a tumour-suppressive response.

\section{DNA Repair}

HOXB7 is one of the HOX genes which shows a markedly higher expression in ovarian cancer cell lines compared to normal ovarian epithelia [15] and promotes growth in ovarian epithelial cells [11], but it also plays a novel role in DNA double-strand break repair through interacting with proteins that act as genomic caretakers, including members of the DNA-dependent protein kinase haloenzyme, Ku70, Ku80 and DNA-PK $\mathrm{Cs}_{\text {[2] }}$ [25. Binding of HOXB7 to such haloenzymes endogenously and exogenously increased DNA repair through poly (ADP) ribose polymerase (PARP) activity. Different HOXB7 expressing breast cancer cell lines exposed to ionising radiation (IR) showed enhanced end-joining product formation and enhanced double-strand break repair and non-malignant cell lines that were transfected with a HOXB7 expression vector developed increased resistance to killing by IR. Correspondingly, chromosomal damage was reduced following IR and less residual damage was seen in cells expressing $H O X B 7$, an effect which could be reversed by $H O X B 7$ silencing. These findings suggest that HOXB7 could be a potential target for therapies that enhance IR cell killing.

\section{Targeting HOX genes}

As the function of the $H O X$ genes is partly based on the binding of HOX proteins to the TALE homeobox set of co-factors, PBX and MEIS, the oncogenic features of the aberrantly expressed HOX proteins could be impaired by targeting these co-factors. PBX and MEIS have been found extensively expressed both nuclear and cytoplasmically in ovarian carcinomas but only MEIS 1 and 2 are expressed in the nucleus of normal epithelia [26]. This suggests that these co-factors are important in ovarian carcinogenesis most probably by potentiating the function of aberrantly expressed HOX proteins.

A peptide called HXR9 has been designed to target the interaction between HOX and PBX. This drug has been shown to retard tumour growth and induce apoptosis in the ovarian cancer cell line SKOV-3 [27], a cell line which shows highly deregulated expression of certain $H O X$ genes. However, this effect was not seen in the OV-90 cell line which does not show HOX gene deregulation. HXR9 treatment has proven to be successful in other malignancies showing $H O X$ gene deregulation including melanoma [28], myeloma [29], renal cancer [30] and lung cancer [31]. These results show that $\mathrm{PBX} / \mathrm{HOX}$ binding is a potential target in ovarian cancer.

\section{HOX genes as potential biomarkers and prognostic markers}

Circulating autologous antibodies to tumour antigens are potential diagnostic biomarkers for the detection of early stage cancers. A systematic search for an ovarian tumour antigen was undertaken by Naora et al (2001) [15]. In this study, the SEREX methodology (serological identification of antigens by recombinant expression cloning) was used to screen tumour cDNA expression libraries with ovarian cancer patient serum. HOXB7 was one of the $H O X$ genes found in this screen, with significant serological reactivity to HOXB7 in 13 of the 39 ovarian cancer patients and in 1 healthy female, suggesting the detection of anti-HOXB7 antibodies could act as a possible diagnostic tool $[13,15]$. However, further research using larger sample number sizes and correlation studies of titre of anti-HOXB7 with disease stage is needed. Similar methodology applied to the serum of patients with serous ovarian carcinomas identified the HOXA7 gene as a potential biomarker of this disease [12], and HOXA10 as a promising prognostic marker for OCCA as its over expression is strongly correlated with poor survival and not expressed in normal OSE [22].

$H O X$ genes showing markedly higher expression in ovarian cancer cell lines and cancer tissue specimens compared to the normal ovaries also have the potential of acting as prognostic markers, these include HOXB4 [16] and HOXB7 [25]. In microarray analysis of ovarian tissues, HOXA5 and HOXA9 were both shown to be overexpressed [32]. HOXA5 has been found to act as a tumour suppressor in breast tissue by transactivating the $p 53$ gene [7] to induce apoptosis by p53-dependent and p53-independent mechanisms [33]. A possible tumour-suppressor role for HOXA5 is also supported by low HOXA5 expression in breast [7] and lung cancer [34] tissues which is thought to be mediated by methylation of the CpG island located on the $5^{\prime}$ end of the HOXA5 gene [7].

In addition to expression profiling, epigenetic alterations associated with ovarian carcinogenesis have been studied [35-37]. The most common molecular alteration in human neoplasia is DNA methylation [38] and this could possibly act as a prognostic marker. Fiegl et al. (2008) [39] analysed the DNA methylation of 71 genes in 22 ovarian cancers and 18 non-neoplastic samples and identified the best discriminators between cancer 
and non-neoplastic tissue as being HOXA10 and HOXA11. In particular HOXA11 methylation was strongly associated with poor outcome, suggesting a possible role for DNA methylation as a prognostic marker in ovarian cancer. A more recent study has shown that increased expression of HOXA10 is present in ovarian carcinomas as a result of promoter hypomethylation of HOXA10 [40]. This could also act as a prognostic factor in ovarian cancer as well as a possible therapeutic target, for example by using drugs that can reverse epigenetic changes.

\section{Conclusions}

$H O X$ gene function is associated with the oncogenesis of ovarian cancer, having a proven role in proliferation, cell migration and DNA repair, although the exact mechanisms and pathways involved require further study. Their oncogenic function together with the observed differences in expression between normal ovarian tissue and ovarian cancers suggest a potential diagnostic and prognostic value for some $H O X$ genes. This potential is broad ranging as the oncogenic and/ or tumour suppressor functions of HOX genes indicate numerous possible influences on the subtype of ovarian cancer, its aggressiveness and the likelihood of metastasis and angiogenesis, together with its response to at least certain types of therapy, including radiotherapy. The expression of individual $H O X$ genes or groups of $H O X$ genes in tumours, circulating cells or cells in the ascites, or the presence of HOX proteins in a range of biological fluids are all potential sources of valuable clinical information.

It is also apparent that $H O X$ genes are potential therapeutic targets, as are the mechanisms which regulate HOX protein function, such as the HOX/PBX heterodimer which upon disruption has shown anti-tumour effects in a variety of cancers $[27,28,30,31]$. These treatments could be used alone or in combination with other treatment modalities to increase tumour susceptibility; for example treating ovarian tumours showing highly elevated levels of HOXB7 with HXR9 could sensitise cells to killing by ionising radiation. This approach may also be valuable for treatment of drug-resistant cancers, especially if $H O X$ expression in the tumour indicates a high level of $H O X$ deregulation.

\section{Acknowledgements}

ZLK is supported by GRACE, a gynaecological cancer charity based in the UK.

\section{Author details}

'Postgraduate Medical School, Faculty of Health and Medical Sciences, University of Surrey, GU2 7WG, UK. ${ }^{2}$ Royal Surrey County Hospital, Egerton Road, Guildford, Surrey, GU2 7XX, UK.

\section{Authors' contributions}

ZLK and RGLM wrote the review. AM, HSP and SBM provided advice on which literature to include and which studies were most relevant. All authors have read and approved the final manuscript.

\section{Competing interests}

The authors declare that they have no competing interests.

Received: 28 June 2011 Accepted: 9 September 2011 Published: 9 September 2011

\section{References}

1. McGinnis W, Krumlauf R: Homeobox genes and axial patterning. Cell 1992, 68(2):283-302.

2. Veraksa A, Del Campo M, McGinnis W: Developmental patterning genes and their conserved functions: From model organisms to humans. Molecular Genetics and Metabolism 2000, 69(2):85-100.

3. Garcia-Fernandez J: The genesis and evolution of homeobox gene clusters. Nature Reviews Genetics 2005, 6(12):881-892

4. Abate-Shen C: Deregulated homeobox gene expression in cancer: Cause or consequence? Nature Reviews Cancer 2002, 2(10):777-785.

5. Calvo R, et al: Altered HOX and WNT7A expression in human lung cancer. Proceedings of the National Academy of Sciences of the United States of America 2000, 97(23):12776-12781.

6. Miller GJ, et al: Aberrant HOXC expression accompanies the malignant phenotype in human prostate. Cancer Research 2003, 63(18):5879-5888.

7. Raman $V$, et al: Compromised HOXA5 function can limit $\mathrm{p} 53$ expression in human breast tumours. Nature 2000, 405(6789):974-978.

8. Vider BZ, et al: Human colorectal carcinogenesis is associated with deregulation of homeobox gene expression. Biochemical and Biophysical Research Communications 1997, 232(3):742-748.

9. Cantile $M$, et al: Hyperexpression of locus $C$ genes in the HOX network is strongly associated in vivo with human bladder transitional cell carcinomas. Oncogene 2003, 22(41):6462-6468.

10. Taylor HS, VandenHeuvel GB, Igarashi P: A conserved Hox axis in the mouse and human female reproductive system: Late establishment and persistent adult expression of the Hoxa cluster genes. Biology of Reproduction 1997, 57(6):1338-1345.

11. Cheng $W$, et al: Lineage infidelity of epithelial ovarian cancers is controlled by HOX genes that specify regional identity in the reproductive tract. Nat Med 2005, 11(5):531-537.

12. Naora $\mathrm{H}$, et al: Aberrant expression of homeobox gene HOXA7 is associated with mullerian-like differentiation of epithelial ovarian tumors and the generation of a specific autologous antibody response. Proceedings of the National Academy of Sciences of the United States of America 2001, 98(26):15209-15214.

13. Yamashita $T$, et al: Suppression of invasive characteristics by antisense introduction of overexpressed HOX genes in ovarian cancer cells. International Journal of Oncology 2006, 28(4):931-938.

14. Ota T, et al: HOXA7 in epithelial ovarian cancer: Interrelationships between differentiation and clinical features. Reproductive Sciences 2007, 14(6):605-614.

15. Naora $H$, et al: A serologically identified tumor antigen encoded by a homeobox gene promotes growth of ovarian epithelial cells. Proceedings of the National Academy of Sciences of the United States of America 2001, 98(7):4060-4065

16. Hong $\mathrm{JH}$, et al: Expression pattern of the class I homeobox genes in ovarian carcinoma. Journal of Gynecologic Oncology 2010, 21(1):29-37.

17. Bodey B, Siegel SE, Kaiser HE: Immunocytochemical detection of the homeobox B3, B4, and C6 gene products in breast carcinomas. Anticancer Research 2000, 20(5A):3281-3286.

18. Zhang $X B$, et al: High incidence of leukemia in large animals after stem cell gene therapy with a HOXB4-expressing retroviral vector. Journal of Clinical Investigation 2008, 118(4):1502-1510.

19. Bodey B, et al: Homeobox B3, B4, and C6 gene product expression in osteosarcomas as detected by immunocytochemistry. Anticancer Research 2000, 20(4):2717-2721.

20. Bodey B, et al: Immunocytochemical detection of homeobox B3, B4, and $\mathrm{C} 6$ gene product expression in lung carcinomas. Anticancer Research 2000, 20(4):2711-2716. 
21. Miao J, et al: HOXB13 promotes ovarian cancer progression. Proceedings of the National Academy of Sciences 2007, 104(43):17093-17098.

22. Li B, et al: HOXA10 is Overexpressed in Human Ovarian Clear Cell Adenocarcinoma and Correlates With Poor Survival. International Journal of Gynecological Cancer 2009, 19(8):1347-1352.

23. Ota T, et al: Expression and function of HOXA genes in normal and neoplastic ovarian epithelial cells. Differentiation 2009, 77(2):162-171.

24. Klausen C, Leung PCK, Auersperg N: Cell Motility and Spreading Are Suppressed by HOXA4 in Ovarian Cancer Cells: Possible Involvement of beta 1 Integrin. Molecular Cancer Research 2009, 7(9):1425-1437.

25. Rubin $\mathrm{E}$, et al: A role for the HOXB7 homeodomain protein in DNA repair. Cancer Research 2007, 67(4):1527-1535.

26. Crijns APG, et al: MEIS and PBX homeobox proteins in ovarian cancer. European Journal of Cancer 2007, 43(17):2495-2505.

27. Morgan $\mathrm{R}$, et al: Targeting $\mathrm{HOX}$ and $\mathrm{PBX}$ transcription factors in ovarian cancer. BMC Cancer 2010, 10(1):89.

28. Morgan R, et al: Antagonism of HOX/PBX dimer formation blocks the in vivo proliferation of melanoma. Cancer Research 2007, 67(12):5806-5813.

29. Daniels TR, et al: Disruption of HOX activity leads to cell death that can be enhanced by the interference of iron uptake in malignant $B$ cells. Leukemia 2010, 24(9):1555-65.

30. Shears $\mathrm{L}$, et al: Disrupting the Interaction Between HOX and PBX Causes Necrotic and Apoptotic Cell Death in the Renal Cancer Lines CaKi-2 and 769-P. Journal of Urology 2008, 180(5):2196-2201.

31. Plowright $L$, et al: HOX transcription factors are potential therapeutic targets in non-small-cell lung cancer (targeting HOX genes in lung cancer). British Journal of Cancer 2009, 100(3):470-475.

32. Bahrani-Mostafavi Z, et al: Correlation Analysis of HOX, ErbB and IGFBP Family Gene Expression in Ovarian Cancer. Cancer Investigation 2008, 26(10):990-998.

33. Chen H, Chung S, Sukumar S: HOXA5-Induced Apoptosis in Breast Cancer Cells Is Mediated by Caspases 2 and 8. Mol Cell Biol 2004, 24(2):924-935.

34. Shiraishi $\mathrm{M}$, et al: HOX gene clusters are hotspots of de novo methylation in CpG islands of human lung adenocarcinomas. Oncogene 2002, 21(22):3659-3662.

35. Teodoridis JM, et al: CpG island methylation of DNA damage response genes in advanced ovarian cancer. Cancer Research 2005, 65(19):8961-8967.

36. Muller HM, et al: Analysis of methylated genes in peritoneal fluids of ovarian cancer patients: A new prognostic tool. Clinical Chemistry 2004, 50(11):2171-2173.

37. Wei $\mathrm{SH}$, et al: Prognostic DNA methylation biomarkers in ovarian cancer. Clinical Cancer Research 2006, 12(9):2788-2794.

38. Laird PW: The power and the promise of DNA methylation markers. Nature Reviews Cancer 2003, 3(4):253-266.

39. Fiegl $\mathrm{H}$, et al: HOXA11 DNA methylation-A novel prognostic biomarker in ovarian cancer. International Journal of Cancer 2008, 123(3):725-729.

40. Cheng W, et al: Identification of aberrant promoter hypomethylation of HOXA10 in ovarian cancer. Journal of Cancer Research and Clinical Oncology 2010, 136(8):1221-1227.

doi:10.1186/1757-2215-4-16

Cite this article as: Kelly et al: HOX genes in ovarian cancer. Journal of Ovarian Research 2011 4:16.

\section{Submit your next manuscript to BioMed Central and take full advantage of:}

- Convenient online submission

- Thorough peer review

- No space constraints or color figure charges

- Immediate publication on acceptance

- Inclusion in PubMed, CAS, Scopus and Google Scholar

- Research which is freely available for redistribution

Submit your manuscript at www.biomedcentral.com/submit
Biomed Central 